\title{
Studies of different numerical models for a turbulent particulate flow in a square pipe with $90^{\circ}$ bend
}

\author{
D. Schellander, D. Kahrimanovic \& S. Pirker \\ Christian Doppler Laboratory on Particulate Flow Modelling, \\ Johannes Kepler University, Austria
}

\begin{abstract}
In process industry simulating particulate flow is one of the actual top level research topics. Driven by the wish for readily available simulation results with predictive description of the processes in fluid and granular flow, many mathematical models have been proposed.

Among these models Eulerian and Lagrangian based particle models are most common. These models have been additionally augmented by sub-models in order to picture effects like particle rotation, rough wall treatment or interparticle collisions. The last one includes in Lagrangian phase a very high increase of computational effort because of finding collision partners.

In this paper these models are tested at a very simple flow situation of a $90^{\circ}$ bended square duct flow. From classical kinetic theory, Eulerian particle model is considered and also a discrete phase model, known as Lagrangian particle model. Finally, a hybrid Eulerian-Lagrangian model, EUgran+, is introduced using a new radial distribution coefficient, based on measurements of Gidaspow and Huilin. The presented hybrid model is based on an Eulerian model that is augmented by Lagrangian tracer particles. These tracer particles provide additional information on e.g. particle rotation and particle wall treatment that prepares information for the classical Eulerian phase, using coupling equations.

It was the aim to create a robust simulation model for simulating particulate flows with high and low loaded particle regions. The second aim was to create a model which gives comparable accuracy as a Lagrangian model with particle collision, without calculating collisions in Lagrangian phase, to decrease the computational time.
\end{abstract}

Keywords: hybrid model, particulate flow, transport system, tracer particles. 


\section{Introduction}

The pneumatic transport and separation of materials, like dust, sand, cement, coal and other particle based granular materials is in common use in the industry. It can be found in the chemical industry, the coal industry, cement industry and many other industries. Pulverised material is transported from one place in the company to another place using pneumatic conveying systems with pipes, containing elbows, bends and cyclones.

Since computer simulation is in our mind, scientists dream of simulating particulate flows, but there were and are already limitations. For particle interaction and flow simulation many equations are known, but nowadays it is not possible to calculate the flow of an arbitrary geometry exactly. Computers are nowadays more powerful than ten years ago but also today it is not possible to simulate realistic high loaded particle conveying systems. These high loaded regions are in a standard cyclone present and should be simulated.

For the simulation of particles in a fluid, two main models are now state of the art. Thus we firstly present the Discrete Phase Model (DPM) and the Eulerian Model (EM). The DPM describes single particles on their way through the simulation region. The EM describes the particles as a continuous phase, introducing a granular temperature. The granular temperature is a value for the particle velocity fluctuation (e.g. Dartevelle [1]). Both models have their advantages and disadvantages, especially in regions with low and high particle loadings.

Secondly our Hybrid-Model (HM) is presented and depicted in detail, called EUgran+ (e.g. Pirker et al. [2]). There are other hybrid models too, for example the Dense Discrete Phase Model (DDPM), described in e.g. Fluent [3] which is based on Lagrangian particle trajectories which get information from the Eulerian phase. The EUgran+ model is based on the Eulerian granular phase and is modified by information from Lagrangian tracer particles.

\section{Fluid phase}

The fluid phase is described by the Navier-Stokes equations. These equations are state of the art and used in Computational Fluid Dynamics (CFD) to describe a fluid flow. With additional models, like the Reynold Average Navier-Stokes equations, turbulence can be modelled (e.g. Fluent [3])

$$
\begin{gathered}
\frac{\partial}{\partial t}\left(\alpha_{g} \rho_{g}\right)+\nabla \cdot\left(\alpha_{g} \rho_{g} \mathbf{u}_{g}\right)=\mathbf{0}, \\
\frac{\partial}{\partial t}\left(\alpha_{g} \rho_{g} \mathbf{u}_{g}\right)+\nabla \cdot\left(\alpha_{g} \rho_{g} \mathbf{u}_{g} \mathbf{u}_{g}\right)= \\
-\alpha_{\mathbf{g}} \nabla p_{g}+\nabla \cdot \alpha_{g} \tau_{g}+\mathbf{f}_{g, d r a g}+\mathbf{f}_{g, a d d} .
\end{gathered}
$$




\section{Discrete phase model}

\subsection{Introduction}

The standard Lagrangian DPM is based on a translational force balance

$$
\frac{\partial}{\partial t} \mathbf{u}_{p}=\frac{18 \mu_{g}}{\rho_{p} d_{p}^{2}} \frac{C_{D} \operatorname{Re}_{p}}{24}\left(\mathbf{u}_{g}-\mathbf{u}_{p}\right)+\mathbf{g}+\mathbf{f}_{p, a d d} .
$$

It can be seen in this equation that all forces in the Lagrangian phase are based on the mass of one particle. Additionally in this paper an angular momentum balance is used, to account for particle rotation and Magnus force calculations.

$$
\frac{d \boldsymbol{\omega}_{p}}{d t}=\mathbf{t}_{p, g}+\mathbf{t}_{p, a d d}
$$

\subsection{Additional forces}

\subsubsection{Magnus force and torque}

The standard DPM neglects the force created by particle rotation, the Magnus force, which was detected by Newton first. Here the acceleration of one particle, induced by the Magnus force is shown (e.g. Kahrimanovic [4]).

$$
\mathbf{f}_{p, \text { Magnus }}=\frac{1}{2}\left|\mathbf{u}_{g}-\mathbf{u}_{p}\right| C_{L} r_{p}^{2} \pi\left(\frac{\Omega_{p} \times\left(\mathbf{u}_{g}-\mathbf{u}_{p}\right)}{\Omega_{p}}\right) \frac{\rho_{g}}{m_{p}}
$$

The Magnus force describes a force induced by rotation of a particle, because of different pressure regions within the boundary layer around the particle.

$$
\Omega_{p}=\frac{1}{2} \nabla \times \mathbf{u}_{g}-\omega_{p}
$$

(6) describes the difference between the rotating fluid and the particle rotation, $C_{L}$ is the lift coefficient (e.g. Lun and Lui [5]).

For the calculation of the angular momentum balance, the fluid-torque and additional torque from interparticle collisions is needed. The fluid-torque can be written as (e.g. Pirker et al. [2])

$$
\mathbf{t}_{p, g}=\frac{\mu_{g}}{64 I_{p}} d_{p}^{3} C_{R} \Omega_{p} \operatorname{Re}_{R}
$$

with $\operatorname{Re}_{R}$ as rotating Reynolds-number and $I_{p}$ as value in the main diagonal of the inertia tensor.

$$
I_{p}=\frac{m_{p} d_{p}^{2}}{10}, \quad \operatorname{Re}_{R}=\frac{\rho_{g} d_{p}^{2}\left|\boldsymbol{\Omega}_{p}\right|}{\mu_{g}}
$$


The rotation coefficient (e.g. Sommerfeld [6]) is written as

$$
C_{R}=\left\{\begin{array}{ccc}
\frac{64 \pi}{\mathrm{Re}_{R}} & \ldots & \mathrm{Re}_{R}<32 \\
\frac{12.9}{\sqrt{\mathrm{Re}_{R}}+\frac{128.4}{\mathrm{Re}_{R}}} & \ldots & 32 \leq \mathrm{Re}_{R}<1000
\end{array}\right.
$$

Collisional forces between particles in a strand effects that the rotation of the particles decreases. The rotational energy is transformed via friction into temperature, also granular temperature which is neglected in this paper, because of the very small values. The following equation describes the interaction between collisions and angular velocity changing in one timestep.

$$
\mathbf{t}_{p, a d d}=-C_{t} N_{s, p} \frac{\boldsymbol{\omega}_{p}}{t_{t p}}
$$

The number of collisions in one timestep can be calculated as

$$
N_{s, p}=n_{s}^{2} d_{p}^{2} g_{0} \sqrt{\pi \Theta_{s}} \frac{t_{t p}}{V_{c}},
$$

with assumption that Eulerian phase and Lagrangian phase represents identical materials (e.g. Kahrimanovic [4], Fluent [3]). $C_{t}$ is the torque factor which can be used to adjust the simulation. In this paper $C_{t}=1$.

\subsubsection{Particle-wall interaction}

Particle-Wall interaction has a very high impact on the result of the simulation. So a well constructed model must be used. For the simulations in this paper a model depicted in Kahrimanovic [4] and Sommerfeld et al. [7] is taken. It can handle rough walls in a sense that the computational effort is in an acceptable range.

\section{Eulerian phase model}

The Eulerian granular phase model, in contrast to the Lagrangian model, considers the multitude of the particles as an artificial continuous solids phase. The individual values of particles are smeared out, the solid phase is based on mean values. The standard Eulerian granular model relies on the assumption that by inter-particle collisions energy is transferred from the mean flow towards chaotic fluctuations of the particles. To describe this behaviour an additional transport equation for the granular temperature must be solved (e.g. Fluent [3] and literature cited therein). 
The main part of this paper is the hybrid model, this gives additional information for the momentum equation, which is written as

$$
\begin{gathered}
\frac{\partial}{\partial t}\left(\alpha_{s} \rho_{s} \mathbf{u}_{s}\right)+\nabla \cdot\left(\alpha_{s} \rho_{s} \mathbf{u}_{s} \mathbf{u}_{s}\right)= \\
-\alpha_{\mathbf{s}} \nabla p_{s}+\nabla \cdot \alpha_{s} \tau_{s}+\alpha_{s} \rho_{s} \mathbf{g}+\mathbf{f}_{s, \text { drag }}+\mathbf{f}_{s, a d d}
\end{gathered}
$$

with the drag force between fluid and solid as

$$
\mathbf{f}_{s, \text { drag }}=K_{s g}\left(\mathbf{u}_{g}-\mathbf{u}_{s}\right)
$$

\subsubsection{Particle-wall interaction}

The particle-wall interaction is based on the same method as mentioned in section 3.1.2.

\section{Hybrid model: EUgran+}

\subsection{Additional forces for Eulerian granular phase and Lagrangian phases}

For the exchange between the Eulerian and Lagrangian phase some additional equations are needed (e.g. Pirker et al. [2]). This must be done because the standard Eulerian granular model lacks some important physics. This includes particle rotation and therefore Magnus force and also the effects of wall roughness. A term describing the force between the two phase caused by different velocities of the Eulerian granular and Lagrangian phase is added. In Fig. 1 the hybrid model EUgran+ is shown in a modular way, so that the different modules and the interaction between them are displayed.

\subsubsection{Dragforce between Eulerian and Lagrangian phase}

Between the Eulerian and Lagrangian phase a dragforce is designed. This force is added to the Lagrangian phase and Eulerian phase.

$$
\mathbf{f}_{p, \text { drag }}=\frac{3\left(1+e_{p, s}\right)}{2 d_{p}} \alpha_{s} g_{0}\left|\mathbf{u}_{s}-\mathbf{u}_{p}\right|\left(\mathbf{u}_{s}-\mathbf{u}_{p}\right)
$$

The equation is based on the force of solid-solid exchange for two different solids. The solid-solid exchange coefficient (e.g. Fluent [3]) for two different solids $l$ and $s$ is written as

$$
K_{l s}=\frac{3\left(1+e_{l s}\right)\left(\frac{\pi}{2}+C_{f r, l s} \frac{\pi^{2}}{8}\right) \alpha_{s} \rho_{s} \alpha_{l} \rho_{l}\left(d_{l}+d_{s}\right)^{2} g_{0, l s}}{2 \pi\left(\rho_{l} d_{l}^{3}+\rho_{s} d_{s}^{3}\right)}\left|\mathbf{u}_{s}-\mathbf{u}_{p}\right|
$$

In this paper only one diameter and density for particles is used, this changes the equation to the shown form (14). The radial distribution coefficient $g_{0}$ which was evaluated from Carnahan and Starling [13], Lun and Savage [14], Sinclair and Jackson [15] and Gidaspow [16], is calculated in a new way. 


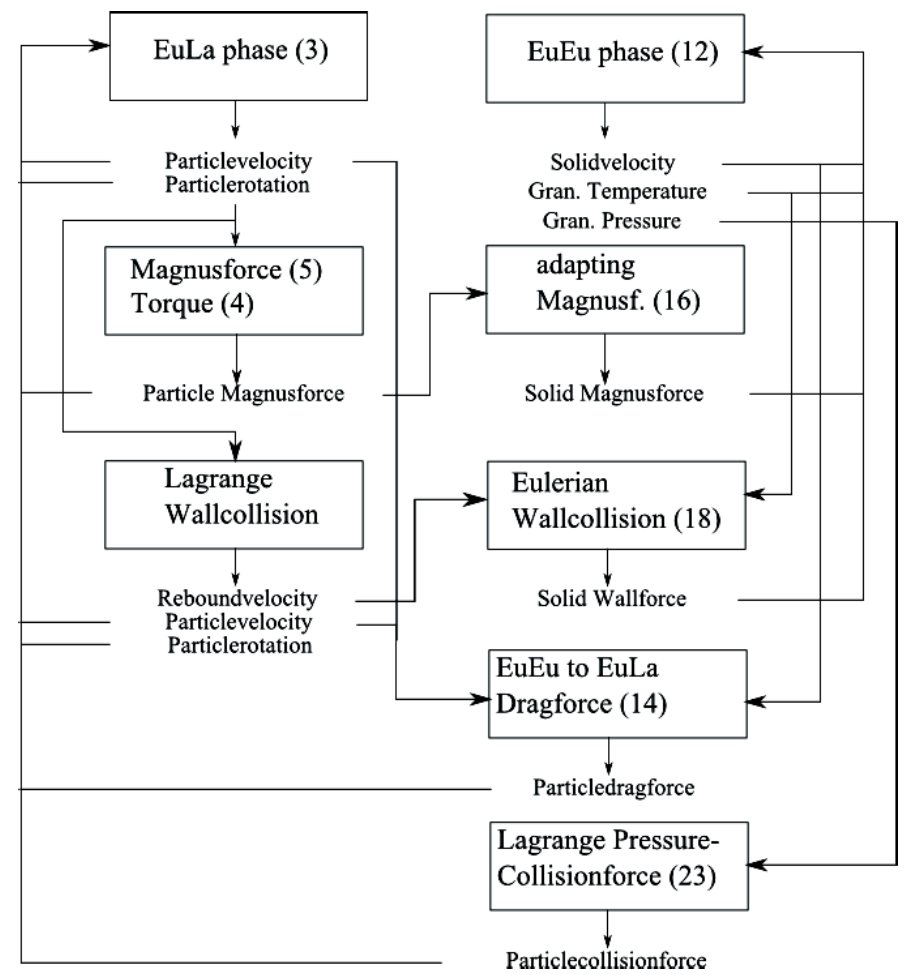

Figure 1: $\quad$ Simple flowchart of the hybrid model; the number in brackets refer to the corresponding equations in the text.

We propose to combine the equations from Gidaspow, Sinclair and Jackson. At the edges the results of both compared to experiments and computational developments, seem to be good but in the middle an enhancement seems to be possible.

$$
g_{0}=\left\{\begin{array}{ccc}
1+\frac{23}{2} \alpha_{s} & \ldots & \alpha_{s}<0.07 \\
\frac{121}{100}+\frac{17}{2} \alpha_{s} & \ldots & 0.07 \leq \alpha_{s}<0.46 \\
\frac{3}{5}\left[1-\left(\frac{\alpha_{s}}{0.67}\right)^{1 / 3}\right]^{-1} & \ldots & 0.46 \leq \alpha_{s}
\end{array}\right.
$$

For this work, (15), was created using the measured-data from Gidaspow and Huilin and experiment-data from Alder and Wainright (e.g. van Wachem et al. [8]) and used for the HM simulations. 


\subsubsection{Magnus force}

The Magnus force, calculated using Lagrangian tracer particles, can be adjusted for the Eulerian phase for each cell with equation

$$
\mathbf{f}_{s, \text { Magnus }}=\alpha_{s} \rho_{s}\left\langle\mathbf{f}_{p, \text { Magnus }}\right\rangle
$$

so that the same acceleration as of one representative particle is added to the solid phase. The average Magnus force in a cell is calculated at each timestep of each tracer particle. The particle timestep for Lagrangian tracer particles is not the same as the simulation timestep. For the $n$-th timestep of all particles hitting one cell

$$
\left\langle\mathbf{f}_{p, \text { Magnus }}\right\rangle_{n}=\frac{(n-1)\left\langle\mathbf{f}_{p, \text { Magnus }}\right\rangle_{n-1}+\mathbf{f}_{p, \text { Magnus }, n}}{n}
$$

is found.

\subsubsection{Wallforce}

For the interaction between wall and solid phase, as mentioned before, a restitution coefficient model is used. Additionally to them a wall force from Lagrangian part is added in the momentum equation (12). Based on the difference of velocities from Lagrangian and Eulerian phase after wall reflection

$$
\mathbf{f}_{s, \text { wall }}=\alpha_{s} \frac{\rho_{s} V_{\text {rebound }}}{V_{c} t_{t s}}\left(\left\langle\mathbf{u}_{p, \text { rebound }}\right\rangle-\left[\mathbf{u}_{s}+\left\langle\mathbf{u}_{s, \text { rebound }}^{\prime}\left(\Theta_{s}\right)\right\rangle\right]\right)
$$

is found. Equation (18) represents a force from the Lagrangian phase to the Eulerian phase. It is assumed that all particles within the volume

$$
V_{\text {rebound }}=u_{s, \perp} t_{t s} A_{c}
$$

collide with the wall during the next timestep. This includes all particles with a velocity higher than

$$
u_{s, \perp}\left(\Theta_{s}\right)=\left|\sqrt{\Theta_{s}} \mathbf{e}_{n}\right|
$$

which is the velocity normal to the wall, and calculated with some assumptions from the granular temperature. It is known that (e.g. Sommerfeld et al. [7])

$$
\Theta_{s}=\frac{u_{s}^{\prime 2}+v_{s}^{\prime 2}+w_{s}^{\prime 2}}{3}
$$

and additionally assumed $u^{\prime}=v^{\prime}=w^{\prime}$. With this knowledge the rebound velocity (22) and the normal velocity (20) to the wall can be estimated.

$$
\mathbf{u}_{\text {s, rebound }}\left(\Theta_{s}\right)=\sqrt{\Theta_{s}} \mathbf{e}_{s, \text { rebound }}
$$

\subsubsection{Collisional force from granular pressure}

If many particles are in one cell then additionally particles can be reflected or captured. This should be modelled with the additional collisional force based on the granular pressure gradient (e.g. Pirker et al. [2]) 


$$
\mathbf{f}_{p, \text { coll }}=-C_{p} \frac{1}{\rho_{p} \alpha_{s}} \nabla p_{s}
$$

with $C_{p}$ as collisional pressure coefficient. $C_{p}$ is used to adapt the strength of the force and in this paper chosen with 1.

\section{Simulations and results}

\subsubsection{Simulation}

For the simulations a simple geometry, a square duct with $90^{\circ}$ bend as depicted in Fig. 2. is taken. The simulations conditions are presented in Tab 1.

Table 1: $\quad$ Simulation parameter.

\begin{tabular}{|c|c|}
\hline Parameter & Value \\
\hline Velocity at inlet $v_{i n}$ & $10 \mathrm{~m} / \mathrm{s}$ \\
\hline Mass loading $L$ & 0.00206 \\
\hline Density fluid $\rho_{g}$ & $1.18 \mathrm{~kg} / \mathrm{m}^{\wedge} 3$ \\
\hline Density particles $\rho_{p}=\rho_{s}$ & $2500 \mathrm{~kg} / \mathrm{m}^{\wedge} 3$ \\
\hline Particle diameter $d_{p}=d_{s}$ & $77 \mu \mathrm{m}$ \\
\hline Mass-Flow rate $\dot{M}_{p}=\dot{M}_{s}$ & $0.000547 \mathrm{~kg} / \mathrm{s}$ \\
\hline
\end{tabular}

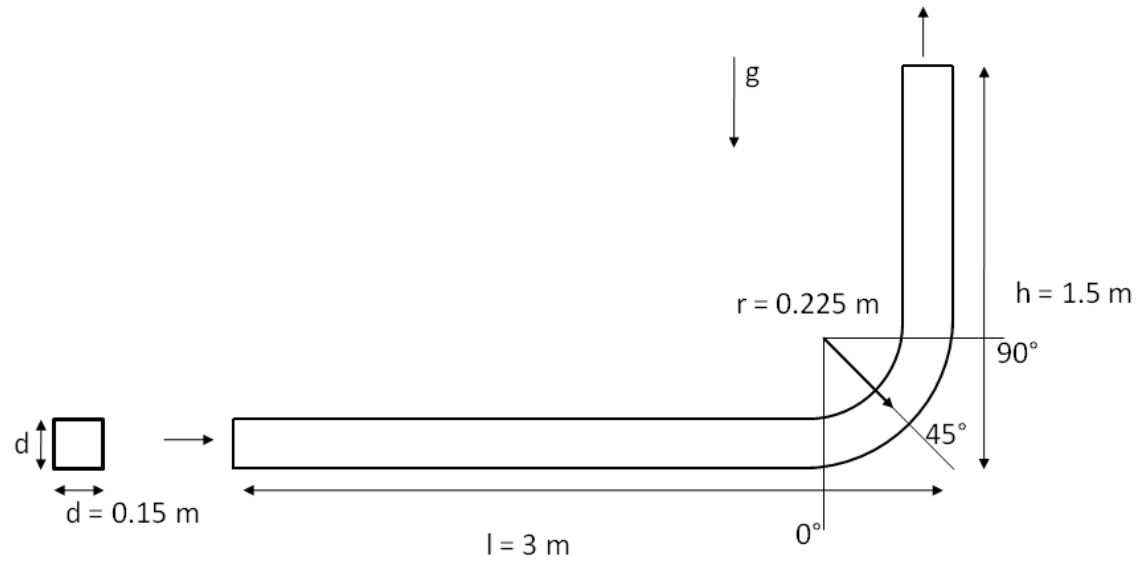

Figure 2: Geometry for simulation with approx. 350000 cells.

For this geometry, (e.g. Mohanarangam et al. $[9,10]$ and Kuan et al. [11]) simulations and experiments are done and described. For this work we decided to use this geometry, because there is known data for comparing our models with 
the experimental data. It should be recognized that the EUgran+ is designed for high loaded particle simulations, which is not the case here.

\subsubsection{Results}

The calculated velocities of the Lagrangian tracer particles and the granular phase are plotted at 3 positions in the duct bend, exactly at $0^{\circ}, 45^{\circ}$ and $90^{\circ}$, which is shown in Fig. 2. Additionally the measured data is depicted. In Fig. 3 the comparison of the different granular velocities can be seen. The Eulerian phase is only present at the cells which are nearest to the wall, which gives very bad results. The modelled Lagrangian phase (e.g. Kahrimanovic [4]) gives very good results, in regions which are reached from Lagrangian particles. The Lagrangian simulations are done with 3 different particle diameters $77 \mu \mathrm{m}, 125$ $\mu \mathrm{m}$ and $30 \mu \mathrm{m}$ and the average is plotted. The EUgran+ raises the granular phase away from the wall which gives better results and supports the building of particle strands at the Lagrangian part. The results are better as expected, the hybrid model is very close to the results of the Lagrangian model, regarding interparticle collisions.
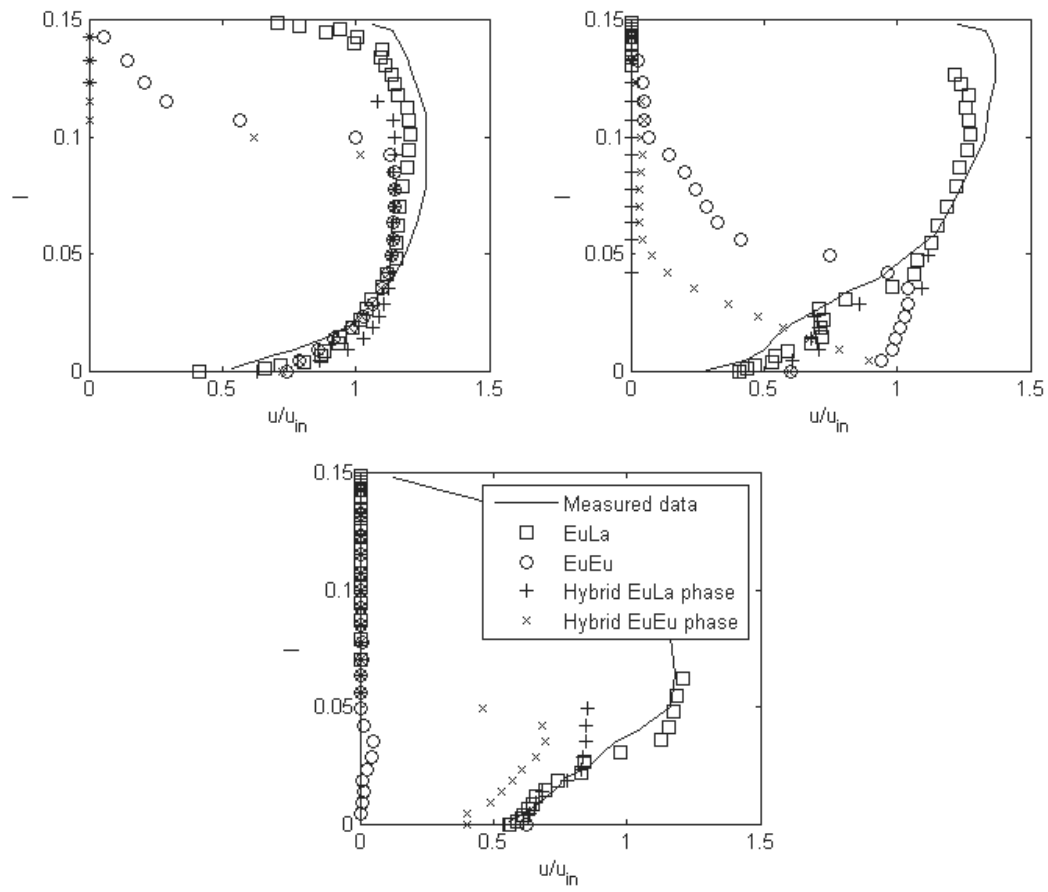

Figure 3: $\quad$ Particle and granular velocities at $0^{\circ}$ (left upper), $45^{\circ}$ (right upper) and $90^{\circ}$ in bend.

Some values in Fig. 3 are zero, because there is no particle or granulate phase at this position for particles with diameter $77 \mu \mathrm{m}$. $l$ is the distance from the 
outer wall in the bend and $u / u_{\text {in }}$ is the average velocity at this point based on the velocity at the inlet.

\section{Conclusion and outlook}

The results with the Lagrangian model (e.g. Kahrimanovic [4]), which has a very high computational effort are very good, compared to the experimental data (e.g. Mohanarangam et al. [9, 10] and Kuan et al. [11]). The computational effort is increasing very strong, with additional particles. This runs into a computational limit. The hybrid model is useful as model for simulations with dilute and highly laden particle regimes, compared to the computational effort. The highly complex particle-particle interaction calculation is included in a shorter way, compared to calculation time, in the Eulerian phase. In regions where only a few particles are present the Lagrangian part has the main impact on the particle fluid interaction, in high loaded regimes the Eulerian part has the higher influence to the simulation result. For simulating cyclones and pneumatic conveying transport systems the EUgran+ is a good approach. For future work a wall treatment model for the Eulerian phase, which brings the high level wall treatment from the Lagrangian phase also to the Eulerian phase will be developed. Then the coupling of Eulerian and Lagrangian phase in wall treatment can be deleted, because both do the same.

For future, it would be additionally important to create a smooth exchange field between discrete phase and granular Eulerian phase (e.g. Pirker et al. [12]). In future work we will include this in the hybrid model. This reduces the computational time significantly (e.g. Pirker et al. [12]) and give a more stable simulation.

\section{Abbreviations and notation}

$\begin{array}{lll}\alpha & \text { volume fraction } & 1 \\ \mu & \text { dynamic viscosity } & \mathrm{kg} /(\mathrm{ms}) \\ \rho & \text { density } & \mathrm{kg} / \mathrm{m}^{3} \\ \Theta & \text { granular temperature } & \mathrm{m}^{2} / \mathrm{s}^{2} \\ \tau & \text { shear rate tensor } & \mathrm{kg} /\left(\mathrm{ms}^{2}\right) \\ \omega & \text { angular velocity } & 1 / \mathrm{s} \\ \Omega & \text { particle fluid rotation } & 1 / \mathrm{s} \\ C & \text { constant } & 1 \\ d & \text { diameter } & \mathrm{m} \\ e & \text { restitution coefficient } & 1 \\ \mathbf{f}_{p} & \text { force, Lagrangian phase } & \mathrm{m} / \mathrm{s}^{2} \\ \mathbf{f}_{s} & \text { force, Eulerian phase } & \mathrm{kg} /\left(\mathrm{m}^{2} \mathrm{~s}^{2}\right)\end{array}$




$\begin{array}{lll}g & \text { gravity } & \mathrm{m} / \mathrm{s}^{2} \\ g_{0} & \text { radial distribution } & 1 \\ I & \text { inertia tensor } & \mathrm{kgm}^{2} \\ m & \text { mass } & \mathrm{kg} \\ p & \text { pressure } & \mathrm{Pa} \\ r & \text { radius } & \mathrm{m} \\ \mathrm{Re} & \text { Reynolds number } & 1 \\ t & \text { time } & \mathrm{s} \\ \mathbf{t} & \text { angular momentum } & 1 / \mathrm{s}^{2} \\ \mathbf{u} & \text { velocity } & \mathrm{m} / \mathrm{s} \\ & & \\ \text { add } & \text { additional } & \\ \text { coll } & \text { collision } & \\ g & \text { gas phase } & \\ n & \text { normal vector with length } 1 \\ p & \text { particle, parcel of particles } \\ \text { rebound } & \text { rebound from particle wall collision } \\ s & \text { granular solid phase } \\ t p & \text { timestep } & \\ \perp & \text { normal direction to wall }\end{array}$

\section{References}

[1] Dartevelle, S., Numerical and granulometric approaches to geophysical granular flows, Ph.D. thesis, Michigan Technological University, Department of Geological and Mining Engineering, Houghton, Michigan, 2003

[2] Pirker, S., Kahrimanovic, D., Kloss, C., Popoff, B. \& Braun, M., Simulating coarse particle conveying by a set of Eulerian, Lagrangian and hybrid particle models, Powder Technology 204, 2010

[3] Fluent 6.3 User's Guide, Fluent Inc., 2006

[4] Kahrimanovic D., Numerische Simulation und experimentelle Validierung von Gas-Partikel-Strömungen, Ph.D.-thesis, 2009

[5] Lun, C. K. K. \& Lui, H. S., Numerical Simulation of dilute turbulent gassolid flows in horizontal channels, International Journal of Multiphase Flow, 23, pp. 575 - 605, 1997

[6] Sommerfeld, M., Theoretical and Experimental Modelling of Particulate Flows, Lecture Series 2000 - 06, Karman Institute for Fluid Dynamics, 2000

[7] Sommerfeld, M., Crowe, C. \& Tsuji, Y. Multiphase flows with droplet and particles, CRC Press, 1998 
[8] van Wachem, B. G. M., Schouten, J. C. \& van den Bleek, C. M., Comparative Analysis of CFD Models of Dense Gas-Solid Systems, AIChe Journal Vol 47, No. 5, 2001

[9] Mohanarangam. K., Yang, W., Zhang, H. J., and Tu, J. Y., Effect of particles in a turbulent flow within a $90^{\circ}$ bend, Seventh International Conference on CFD in the Minerals and Process Industries CSIRO, Melbourne, Australia, 2009

[10] Mohanarangam, K., Tian, Z. F. \& Tu, J. Y., Numerical simulation of turbulent gas-particle flow in a $90^{\circ}$ bend: Eulerian-Eulerian approach, Computers and Chemical Engineering, 32, pp. 561-571, 2008

[11] Kuan, B., Yang, W., \& Schwarz, M. P., Dilute gas-solid two-phase flows in a curved $90^{\circ}$ duct bend: CFD simulation with experimental validation, Chemical Engineering Science, 2, pp. 2068-2088, 2007

[12] Pirker, S., Kahrimanovic, D. \& Goniva, C., Improving the applicability of discrete phase simulations by smoothening their exchange fields, Applied Mathematical Modelling, 2010

[13] Carnahan, N. F., and K. E. Starling, Equations of State for Non-Attracting Rigid Spheres, J. Chem. Phys., 51, 635, 1969

[14] Lun, C. K. K., and S. B. Savage, The Effects of an Impact Velocity Dependent Coefficient of Restitution on Stresses Developed by Sheared Granular Materials, Acta Mech., 63, 15, 1986

[15] Sinclair, J. L., and R. Jackson, Gas-Particle Flow in a Vertical Pipe with Particle-Particle Interactions, AIChE J., 35, 1473, 1989

[16] Gidaspow, D., Multiphase Flow and Fluidization, Academic Press, San Diego, 1994 